\title{
PROPUESTA DE GUÍA PARA LA SOCIALIZACIÓN DE PROYECTOS EÓLICOS EN HONDURAS
}

\author{
Rigoberto Rodríguez Ávila, Oscar Andrés Rivera Portillo \\ y Wilfredo César Flores Castro ${ }^{1}$ \\ Facultad de Postgrado, Universidad Tecnológica Centroamericana (UNITEC), \\ Campus Tegucigalpa
}

(Recibido: Octubre, 2017/ Aceptado: Enero, 2018)

\begin{abstract}
Resumen
El presente es un estudio sobre la manera de socializar correctamente en las fases previas, durante y post de la construcción, generación y manejo de un parque eólico. En Honduras la demanda energética está aumentando, por lo que actualmente se está invirtiendo en nuevas tecnologías, una de ellas es la energía eólica. Debido a que estas energías son nuevas no hay un claro manejo social de cómo se debería intervenir y cómo un proyecto de magnitud considerable no afecte de una manera errónea a la población, por lo que es importante tener en cuenta la regulación, tales como el Convenio OIT 169, protección forestal, protección de la fauna, manejo de tierras, entre otros. Por la amplia diversidad cultural de Honduras, es importante realizar socialización con los pueblos o comunidades de la zona donde se realizará el proyecto y así poder invertir sin futuras posibles consecuencia. Con una correcta socialización un proyecto de generación eléctrica tendrá éxito; en Honduras existen casos lamentables de una mala socialización, pero también existen casos exitosos, tal es el proyecto de Cerro de Hula, el cual tuvo una aceptación positiva de la comunidad. Una guía para la socialización de un proyecto eólico tendrá como fin orientar, mejorar las técnicas y manejo, para intervenir exitosamente en dichas comunidades.
\end{abstract}

Palabras Claves: OIT 169, Acuerdo, Energía Eólica, Correcta Socialización, Guía

\begin{abstract}
This is a study on how to correctly guide the pre-phases, during and post phases of the construction, generation and management of a wind project. In Honduras, energy demand is increasing, that is why there are new technologies and one of them is wind energy. Since these energy sources are new, there is no clear social management on how to intervene and the way a project does not affect wrongly affect the population so it is important to focus on regulations such as the OIT 169 Convention, forest protection, wildlife protection, land management and others. Due to the wide cultural diversity of Honduras, it is important to make agreements with the people or communities in the areas where some project will take place and thus invest without possible future consequences.
\end{abstract}

\footnotetext{
${ }^{1}$ Autor para correspondencia. Email: wilfredo.flores@unitec.edu.hn 
With a correct socialization an electricity generation project will succeed. There are regrettable cases of improper socialization in Honduras, but there are also successful cases such as the project in Cerro de Hula, which had a positive acceptance by the community.

Keywords: Wind Energy, Socialization, Guide.

\section{Introducción}

Honduras es un país que posee una amplia diversidad cultural. De la misma manera, dentro de los atractivos con que cuenta el país se encuentra la biodiversidad, lo cual lo convierte en un importante punto de referencia en recursos energéticos renovables. Hay una percepción general de que los proyectos renovables pueden ser una oportunidad para el desarrollo rural.

Honduras cuenta con un alto potencial eólico que puede promover mejores condiciones sociales, económicas y ambientales para el país. Sin embargo, con una mala socialización se puede generar oposición, especialmente en las comunidades y organizaciones de la sociedad civil, debido a que perciben que los proyectos pueden afectar su entorno o que su desarrollo beneficia a una de las partes, por lo general el desarrollador.

El propósito de este trabajo ha sido la realización de una guía para orientar de una forma adecuada un proyecto eólico en el aspecto social como primer enfoque y luego en otros aspectos, tales como económicos y ambientales. Es muy importante tener en cuenta que en Honduras no existe una guía para una socialización en estos proyectos y los que se han construido actualmente han tomado referencias de otros países para el trabajo social.

A la fecha se ha observado que algunas comunidades se han manifestado en contra de algunos proyectos de energía renovable, debido principalmente a que no se consideran incluidas en los procesos de análisis y consulta acerca de los potenciales impactos negativos que se derivan de estos tipos de proyectos, cuando se establecen en su territorio.

Es necesario revisar y mejorar las leyes, tener una guía, políticas públicas y programas que permitan una efectiva gestión e inclusión social de los proyectos eólicos, en las siguientes páginas se podrá entender mejor como es un proyecto eólico hasta llegar al punto de socialización por medio de una propuesta de guía.

Se espera que esta guía sea una herramienta que ayude a mejorar la comunicación entre los actores más importantes en el desarrollo de proyectos eólicos en Honduras.

\section{Energía Renovable en Latinoamérica}

Según los datos de la Agencia Internacional de Energía, las energías renovables ascienden a casi el 29\% del suministro total de la energía primaria en los países de América Latina, cifra relativamente alta en comparación con la cuota del $5.7 \%$ de energías renovables de los países desarrollados. Dentro del sector predomina la energía hidroeléctrica, con un $62 \%$ 
de la cuota total de energías renovables, llegando a elevarse hasta el 90\% en algunos países como Brasil o Paraguay. Además de provocar cierta inseguridad en el suministro eléctrico, las centrales hidroeléctricas han causado daños medioambientales en algunos lugares del continente, como la selva amazónica. Por otro lado, los países latinoamericanos están expuestos a fuertes y constantes vientos que pueden ser aprovechados para la generación de energía eólica. Por ejemplo, se calcula que Brasil podría llegar a los 140,000 MW de energía del viento, seguido de México con 40,000 MW, Colombia con 20,000 MW, Venezuela que podría llegar a producir 10,000 MW. Asimismo, la energía solar también está distribuida por gran parte de las regiones. Y es que la previsión de incremento de la demanda energética del conjunto de países de América Latina para el año 2030 es del 50\%, lo que hace necesario un incremento de la capacidad instalada de generación de más del $20 \%$. De hecho, se estima que en los próximos años la mayor parte de la financiación de energía se destine a proyectos que utilizan fuentes renovables (Canseco, 2010).

\subsection{Energía Eólica en Honduras}

La primera planta de energía eólica instalada en Honduras se encuentra ubicada en Cerro de Hula, Francisco Morazán, entre los municipios de Santa Ana y San Buenaventura, cuenta con 51 aerogeneradores tipo GAMESA G87 de 2MW cada uno. Recientemente la empresa Energía Eólica de Honduras ha ampliado la planta en 24 MW, es decir, 12 nuevas turbinas con un costo de 60 millones de dólares.

El Segundo parque eólico de gran importancia en el país es el parque Eólico de San Marcos de Colón, el cual genera 50 MW con 25 aerogeneradores ubicados en diferentes lugares del municipio. El parque se encuentra ubicado entre las comunidades de los Llanos y Ojo de Agua, la empresa que llevo a cabo el proyecto es Vientos de Electrotecnia, VESA, con costo de inversión de alrededor de 140 millones de dólares (unos 2,800 millones de lempiras).

Tabla 1. Proyectos eólicos en operación comercial y de capital privado en Honduras.

\begin{tabular}{|c|c|c|c|c|}
\hline $\mathbf{N}^{\circ}$ & Empresa Proponente & Nombre del Proyecto & Ubicación & Potencia Estimada \\
\hline 1 & $\begin{array}{c}\text { Energía Eólica de } \\
\text { Honduras S.A } \\
\text { (Mesoamérica) }\end{array}$ & Honduras 2000 & $\begin{array}{c}\text { Cerro de Hula, } \\
\text { Francisco Morazán }\end{array}$ & $125 \mathrm{MW}$ \\
\hline 2 & $\begin{array}{c}\text { Vientos de Electrotecnia } \\
\text { S.A de C.V. }\end{array}$ & $\begin{array}{c}\text { Vientos de } \\
\text { Electrotecnia }\end{array}$ & $\begin{array}{c}\text { San Marcos de } \\
\text { Colón, Choluteca }\end{array}$ & $49.50 \mathrm{MW}$ \\
\hline & & & & $174.50 \mathrm{MW}$ \\
\hline
\end{tabular}

Fuente: Dirección General de Energía, 2016. Proyectos eólicos en operación comercial y de capital privado en Honduras. Disponible en: http://www.miambiente.gob.hn/

A junio del 2015 los proyectos eólicos con permiso de operación y realizando estudios 658.50 MW, eran 22; los proyectos eólicos con contrato de operación y /o estudio de factibilidad aprobado sumaban $650 \mathrm{MW}$, son 13; y, en operación $174.50 \mathrm{MW}$, son 2 proyectos (Flores, W, 2016). 


\subsection{Evaluación de impacto ambiental en Honduras}

Honduras cuenta con bases constitucionales que pueden soportar legalmente a la Evaluación del Impacto Ambiental, EIA. La Constitución de la Republica, en su Artículo 340 declara "de utilidad y necesidad pública, la explotación técnica y racional de los recursos naturales de la nación", si bien no se trata de una disposición literal en cuanto a las EIA, abre la puerta para establecer interés jurídico y fundamento legal para las actuaciones del Estado en la materia. Sobre esta base constitucional, el proceso de Evaluación de Impacto Ambiental en Honduras, formalmente, se concibe a partir de la Declaración Sobre Medio Ambiente y Desarrollo, hecha durante la Cumbre de Río de Janeiro de 1992. El compromiso asumido por Honduras, al igual que por muchos otros países, consistió en incorporar a la EIA como “...un instrumento nacional, respecto de cualquier actividad propuesta que probablemente haya de producir un impacto negativo considerable en el medio ambiente y que esté sujeta a la decisión de una autoridad nacional competente." La manifestación formal de voluntad fue expresada a través de la aprobación en 1993 de la Ley General del Ambiente, que señala a la evaluación de impacto ambiental como de carácter obligatorio (en su Artículo 5) para proyectos, instalaciones industriales o cualquier actividad pública o privada, susceptible de contaminar o degradar el ambiente, los recursos naturales o el patrimonio histórico cultural de la nación. En esta misma ley se definen los lineamientos generales para lo que sería la aprobación del Sistema Nacional de Evaluación de Impacto Ambiental, SINEIA. El Reglamento del SINEIA contempla los procedimientos de ingreso, trámite, participación ciudadana, y emisión de una Licencia Ambiental. No obstante, este reglamento nació con algunas ambigüedades que limitaron su aplicación eficiente. Por ejemplo, no establecía en forma clara los tipos de proyectos o actividades sujetos a una licencia ambiental. De la misma manera, la ambigüedad afectaba también a definiciones, términos y clasificaciones económicas y técnicas. Lo anterior pareciera ser un verdadero "pecado original" que ha llevado a que prácticamente cualquier proyecto o actividad económica pretenda ser objeto del proceso de licenciamiento ambiental en Honduras, desde un negocio de víveres hasta un gran proyecto de infraestructura, lo que ha desnaturalizando e introducido ineficiencias considerables en la aplicación de políticas ambientales.

Talvez el instrumento de política ambiental que ha observado el mayor nivel de desarrollo en Honduras es el de las Áreas Naturales Protegidas. Existen 107 áreas protegidas, de las cuales solamente 57 tienen base legal y son las que componen el actual sistema de áreas protegidas del país. El resto de las áreas (50) están amparadas en el Acuerdo Presidencial 1118-92. De acuerdo a las características de cada área protegida, éstas se han agrupado en nueve categorías de manejo, listadas en la tabla No. 2. 
Tabla 2. Tabla de información de la Administración Forestal del Estado, Corporación Hondureña de Desarrollo Forestal.

\begin{tabular}{|r|l|r|}
\hline \multicolumn{3}{|c|}{ Categorias de Manejo } \\
\hline No. & \multicolumn{1}{|c|}{ Categoria de Manejo } & $\begin{array}{c}\text { No. De Areas } \\
\text { Protegidas }\end{array}$ \\
\hline 1 & Parque Nacional & 18 \\
\hline 2 & Reserva Biologica & 30 \\
\hline 3 & Reserva Marina & 10 \\
\hline 4 & Refugio de Vida Silvestre & 27 \\
\hline 5 & Monumento Natural & 12 \\
\hline 6 & Monumento Cultural & 2 \\
\hline 7 & Reserva Antropologica & 2 \\
\hline 8 & Area de Uso Multiple & 1 \\
\hline 9 & Reserva de Biosfera & 105 \\
\hline
\end{tabular}

Fuente: AFE-COHDEFOR, 2015. Anuario Estadístico forestal. Disponible en: http://icf.gob.hn/wpcontent/uploads/2015/09/Anuario-Forestal-2007.pdf

En conjunto y de acuerdo a datos preliminares, las áreas protegidas (legales y propuestas) cubren una superficie alrededor de 27,000 kilómetros cuadrados, lo que representa aproximadamente el $24 \%$ del territorio nacional. Sin embargo, la superficie de las áreas protegidas con base legal alcanza aproximadamente 19,000 kilómetros cuadrados, representando el $17 \%$ del territorio nacional. En cualquier caso, este porcentaje es de una magnitud considerable relativa a otros países. Es necesario aclarar que los datos sobre la superficie de las áreas protegidas no son muy exactos y se está en proceso de precisar esta información a través del proceso de racionalización del SINAPH (Banco Mundial, 2012).

\subsection{Comunidades originarias en Honduras}

Los indígenas se distribuyen en 2,128 comunidades a lo largo de 15 de los 18 departamentos de Honduras. Los pueblos originarios indígenas son siete: Chorti, Lenca, Miskito, Nahua, Pech, Tawahka y Tolupán. Los afrodescendientes son dos: los Pueblos Negros del Caribe y los Garífunas. Los más grandes en población son los Lenca y los Garífunas (INE, 2012).

Actualmente sólo el $10 \%$ de los indígenas de Honduras tienen títulos de propiedad de sus tierras. No obstante, el territorio demandado por ellos es de aproximadamente $17.8 \%$ de la superficie nacional. La Confederación de Pueblos Autóctonos de Honduras considera que la falta de títulos es el principal problema que atraviesan los indígenas. Mientras, se los presiona a vender sus tierras, sobre todo en áreas de interés turístico (IGWIA, 2010).

Los territorios indígenas se encuentran difuminados con la gran mayoría de las áreas protegidas del país, entre ellas el Corredor Biocultural Mesoamericano, constituido por la Reserva de Biosfera de Río Plátano, el Parque Nacional Patuca y la Reserva Tawahka 
Asangni, que representa la segunda zona de reserva de biodiversidad más importante del continente, después del Amazonas.

Así las áreas protegidas relacionadas con territorios indígenas cubren más de la mitad del territorio total del Departamento de Gracias a Dios. El manejo de las áreas protegidas que se encuentran dentro de tierras indígenas, debe ser realizado en forma conjunta entre los indígenas y el Estado (Honduras, Ley de Propiedad, art. 101).

Tabla 3. Pueblos Indígenas de Honduras, comunidades y población.

\begin{tabular}{|c|c|c|}
\hline \multicolumn{3}{|c|}{ Pueblos Indigenas de Honduras, comunidades y poblacion } \\
\hline Pueblos Indigenas & Numero de Comunidades & Poblacion \\
\hline Tawahka & 7 & 1,500 \\
\hline Pech & 10 tribus & 3,800 \\
\hline Tolupan & 30 tribus & 18,000 \\
\hline Lenca & 2500 & 72,000 \\
\hline Miskitu & 420 & 76,000 \\
\hline Maya Chortí & 52 & 10,600 \\
\hline Garifuna & 47 & 300,000 \\
\hline Isleños de Habla Inglesa & 46 & 80,000 \\
\hline Nahua & 18 & 19,800 \\
\hline
\end{tabular}

Fuente: Palacios, C., 2007. Pueblos indígenas de Honduras. Disponible en http://www.territorioindigenaygobernanza.com/hon_07.html

\section{Metodología}

\subsection{Flujograma de la metodología e instrumento}

El enfoque metodológico se basó en el exploratorio y entrevista. Se visitó a las comunidades cercanas al proyecto eólico de Cerro de Hula, en vista de que no existe en el país una regulación de este tipo y que se considera que este proyecto siguió las mejores prácticas de socialización de un proyecto eólico instalado en el país. Se recopilaron los datos y se procesaron, según el diagrama metodológico mostrado en la Figura No. 1. 


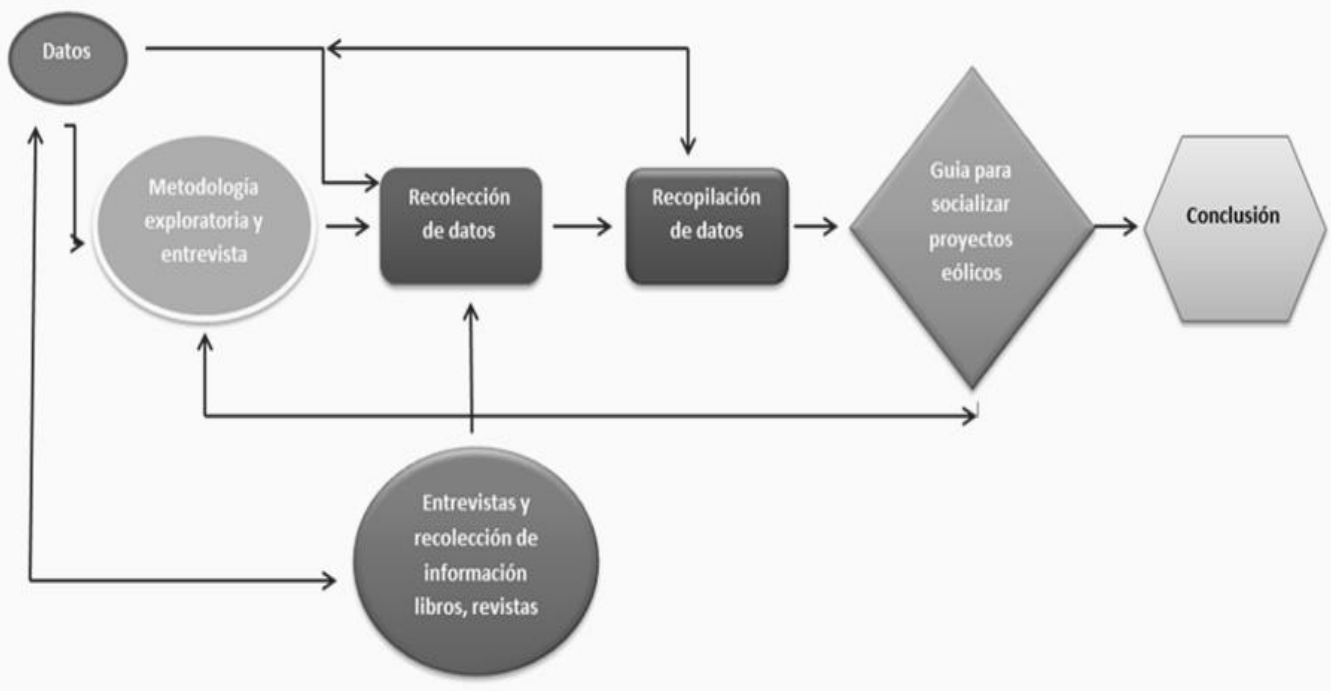

Figura 1. Diseño metodológico

Fuente: Elaboración propia

A continuación, se muestra el instrumento utilizado en la entrevista. Con los resultados de esta entrevista se obtuvieron los datos mostrados en la siguiente sección. El instrumento fue validado por personal de la empresa.

\section{Propuesta de guía para socialización de un proyecto eólico}

Objetivo: Conocer la metodología que se utilizó para socializar el proyecto eólico en el Cerro de Hula.

1. ¿Cómo abordaron a la comunidad donde se ubica el proyecto?

2. ¿Con quién fue el primer acercamiento o los actores que participaron en la socialización del proyecto y cuál fue el paso siguiente que se realizó (se hicieron capacitación, charlas, exposición de los incentivos que se les brindaría, etc.)?

3. ¿Según la empresa cual fue las principales dificultades en la socialización?

4. ¿Se usó algún tipo de Manual o Guía para socializar y si este era de Honduras o extranjero?

5. ¿Con cuántos meses de anticipación se inició la socialización?

6. ¿En su opinión se debería tener una legalización o algún tipo de guía o manual para socializar proyectos de esta índole?

7. ¿Antes, durante y en la actualidad que tipos de incentivos o mejoras a la comunidad se han realizado, se dio trabajo a las personas o como se ayudó para mejorar la economía de la zona?

8. ¿Se realizaron algún tipo de negociaciones con la comunidad en cuanto a terrenos privados y vía pública?

9. ¿En cuánto a la fauna cual ha sido el mayor impacto?

10. ¿Siempre existe una buena comunicación entre la comunidad y el proyecto? 


\subsection{Resultados}

Con la finalidad de crear la guía propuesta de socialización de proyectos eólicos en Honduras, se aplicó una entrevista al personal de la empresa Wind \& Solar del departamento de Ambiente y Desarrollo Social. También se aplicó la entrevista a los pobladores de Ojojona y San Buenaventura. Como resultado a la aplicación de la entrevista a continuación se muestran los resultados más relevantes.

Como se puede observar en la Figura 2, el 50\% de los encuestados dijeron que se abordó el proyecto con la comunidad, con coordinaciones institucionales y especialmente con cada una de las municipalidades de Santa Ana y San Buena Aventura. El 30\% de los encuestados dijo que el abordaje fue con encuestas aplicadas casa por casa y el $20 \%$ con acuerdos de vecinos.

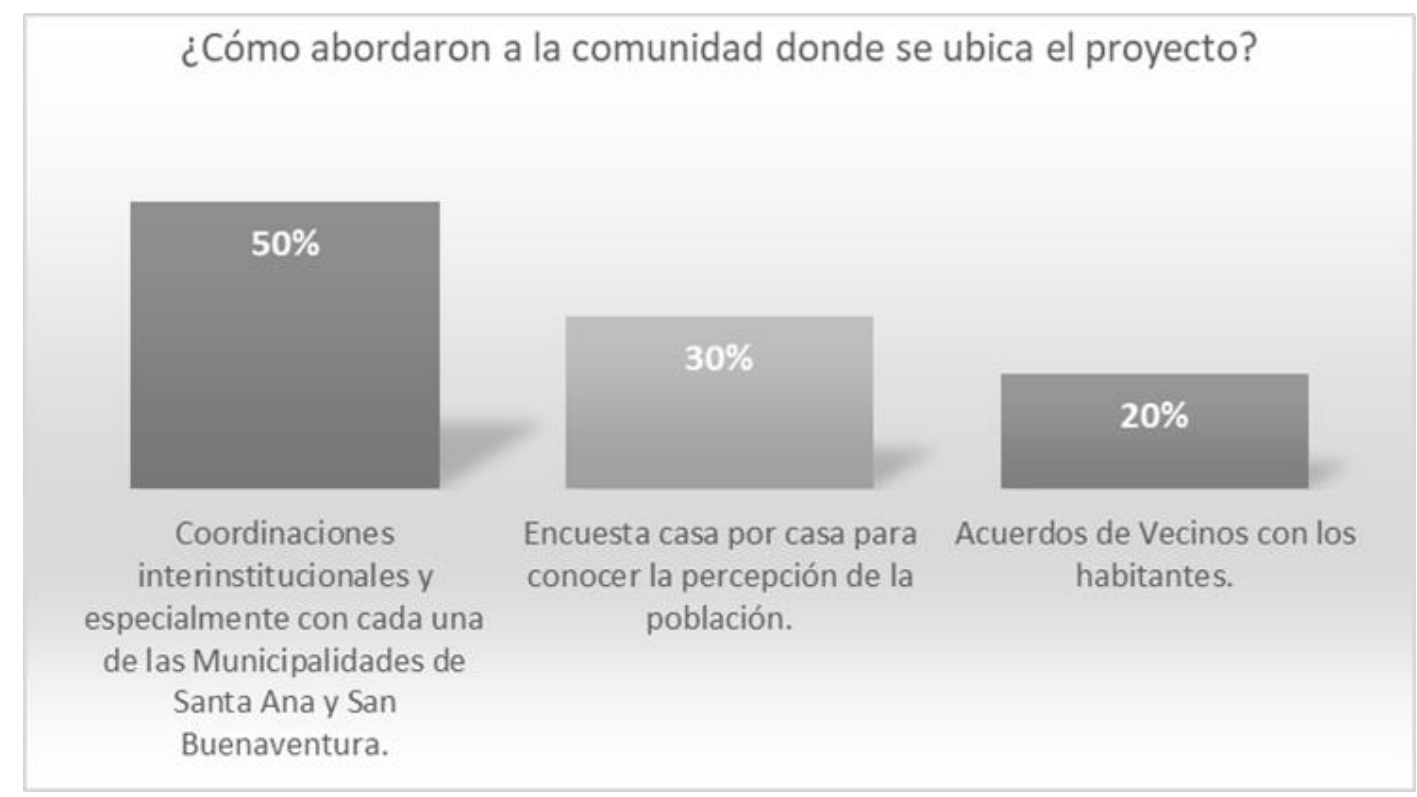

Figura 2. Pregunta \#1

Fuente: Elaboración propia

Según lo observado en el Figura 3, $27 \%$ de las respuestas muestra que las mayores dificultades que se encontró la empresa estaban relacionadas con que éste era el primer parque eólico que se iba a construir en Honduras, y con los arrendamientos de los terrenos, ya que los propietarios de estos no poseían escrituras de los mismos. También, existe una uniformidad de $18 \%$ en explicarle a la comunidad en qué consistía el proyecto y la cantidad de personas que se tenían que informar y el $9 \%$ se encontró que las personas tienen poco nivel educativo. 


\section{Según la empresa ¿Cuál fue la principal dificultad encontrada en la socialización?}

\section{$27 \%$}

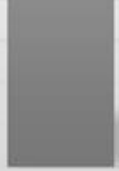

El hecho de que

fuera el primer

parque de este tipo

en el país

representó un reto

importante.

\section{$27 \%$}

$18 \%$

$18 \%$

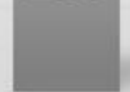

Cantidad de personas que se tenía que informar sobre el proyecto.

\section{$9 \%$}

Arrendamiento de Poco nivel educativo los terrenos

Figura 3. Pregunta \#3

Fuente: Elaboración propia

De la Figura 4 se desprende que el $64 \%$ responde que para la socialización del proyecto no existía guía sobre la socialización, existe una igualdad de $18 \%$ donde se considera que la socialización se hizo de forma presencial y se contactaron con los dueños de los terrenos.

\section{¿Se utilizó algun manual o guía para realizar la socialización?}

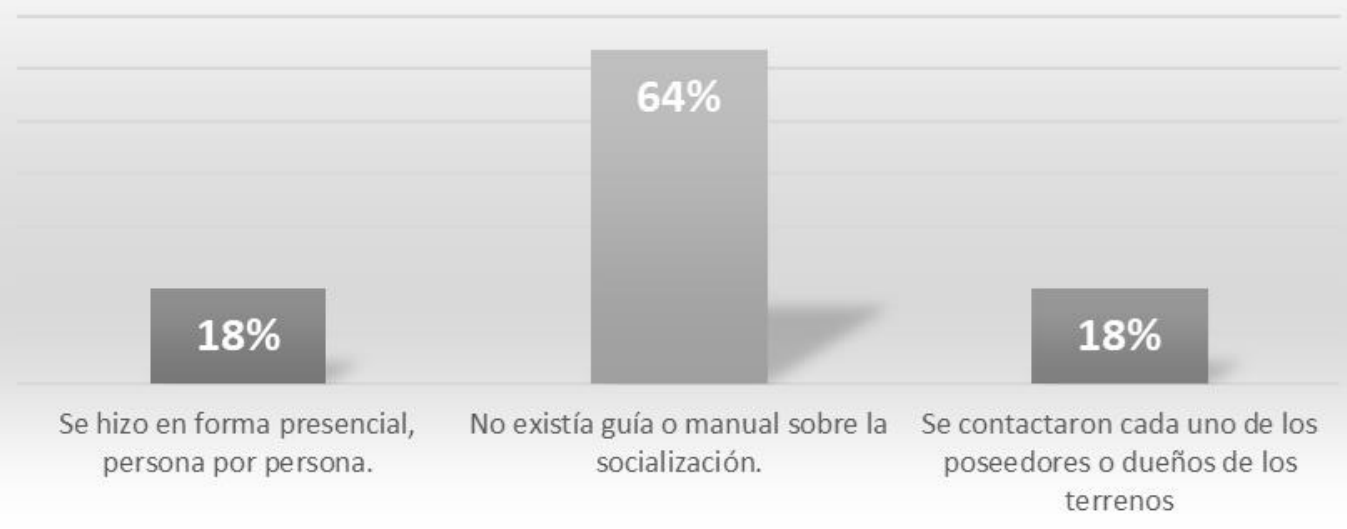

Figura 4. Pregunta \#4

Fuente: Elaboración propia

La Figura 5 muestra que el $78 \%$ de las personas entrevistadas considera que se deben regular los procesos de socialización en los países de la región, con algún tipo de manual o guía y el $22 \%$ discurre que los procesos de consulta y socialización requeridos sean acordes con los impactos ambientales y sociales. 


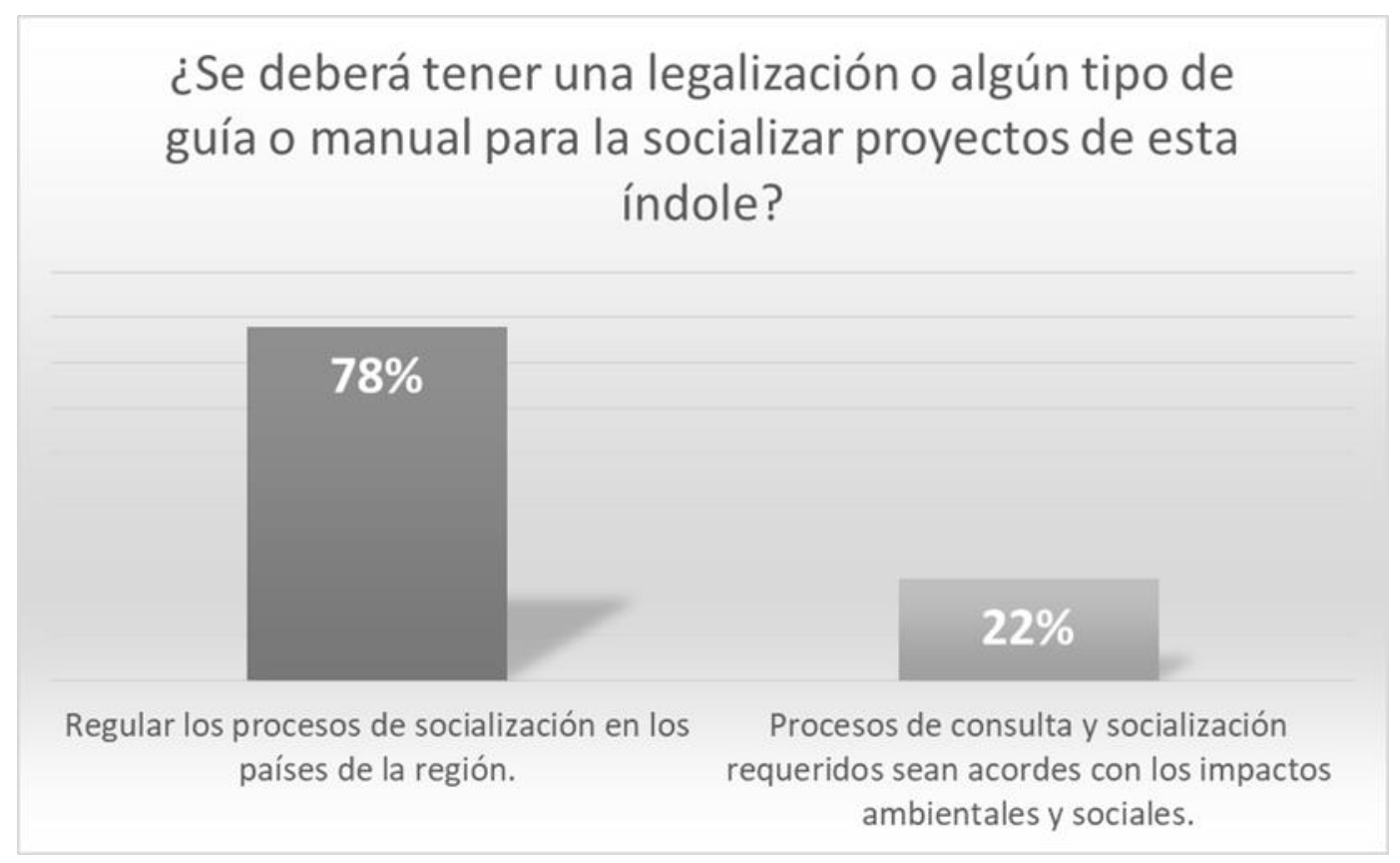

Figura 5. Pregunta \#6

Fuente: Elaboración propia

\subsection{Guía que resultó de la investigación}

A continuación, se muestran los flujogramas que se encuentran en la guía que se obtuvo al aplicar el instrumento antes mostrado.

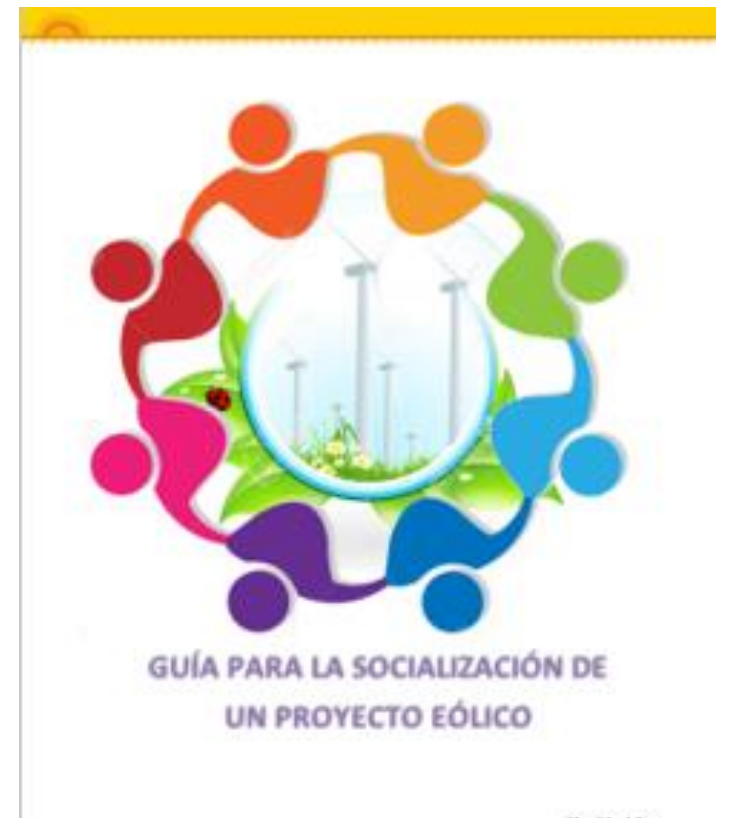

Figura 6. Portada de la Guía

Fuente: Elaboración propia 
Los proyectos pueden crear oportunidades y beneficios para la población, pero al mismo tiempo también pueden tener efectos perjudiciales. En general, nunca son uniformemente buenos o malos, sino que existe una distribución diferencial de costos y beneficios en las comunidades aledañas.

Es necesario llevar a cabo una buena gestión que garantice que los beneficios de los proyectos se maximicen y que se eviten, o minimicen, los impactos negativos de manera constante durante el ciclo del proyecto. La evaluación de impacto social es un proceso que puede contribuir enormemente a garantizar el logro de beneficios y la prevención del daño.

Los impactos sociales también pueden ser causados por proyectos que crean falsas expectativas en una comunidad, ya sea que el personal del proyecto promete intencionalmente y de manera inapropiada cosas que no ocurrirán.

Las comunidades se pueden sentir que se les han mentido cuando los beneficios que han anticipado que recibirían de un proyecto no se concretan. Esta situación contribuye a crear falta de confianza y a la pérdida de la credibilidad de la empresa y el proyecto por lo que provocaría negación de la comunidad.

A continuación, se muestran los pasos que se deberían seguir al momento de socializar un proyecto eólico en Honduras, según lo encontrado en las visitas de campo y en la aplicación del instrumento.

\section{- Antes de la construcción de Proyecto Eólico.}

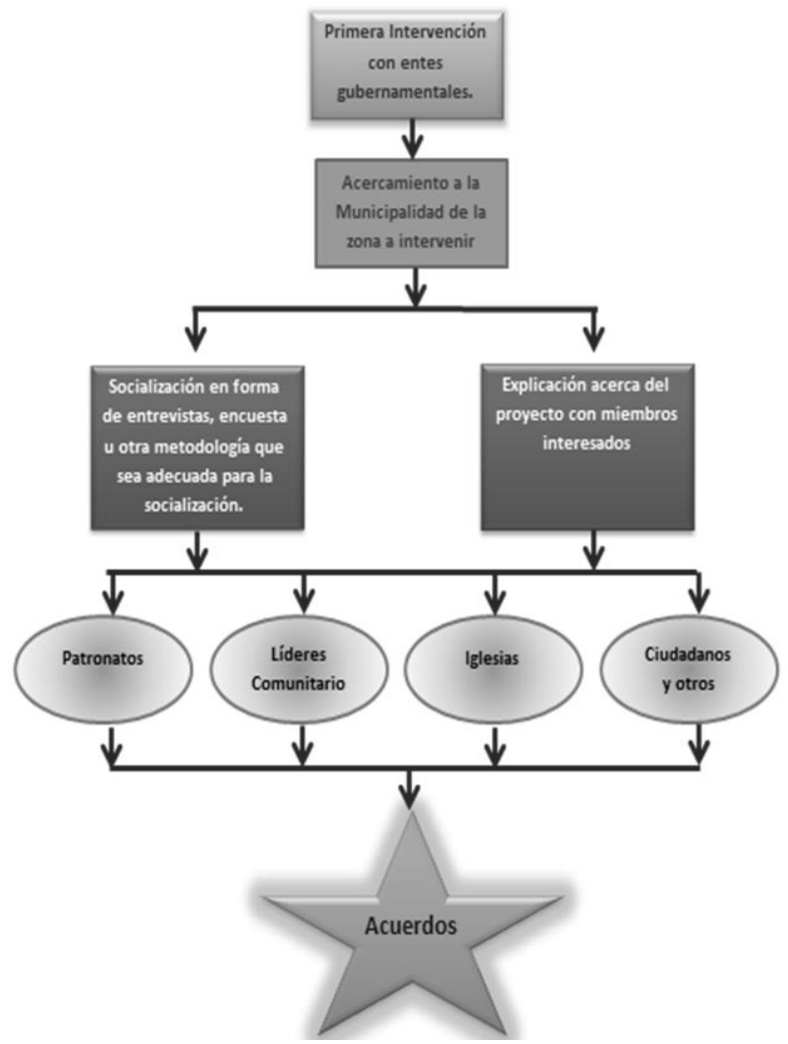

Figura 7. Antes de la construcción de Proyecto Eólico.

Fuente: Elaboración propia 
- Durante la Construcción de un Proyecto Eólico.

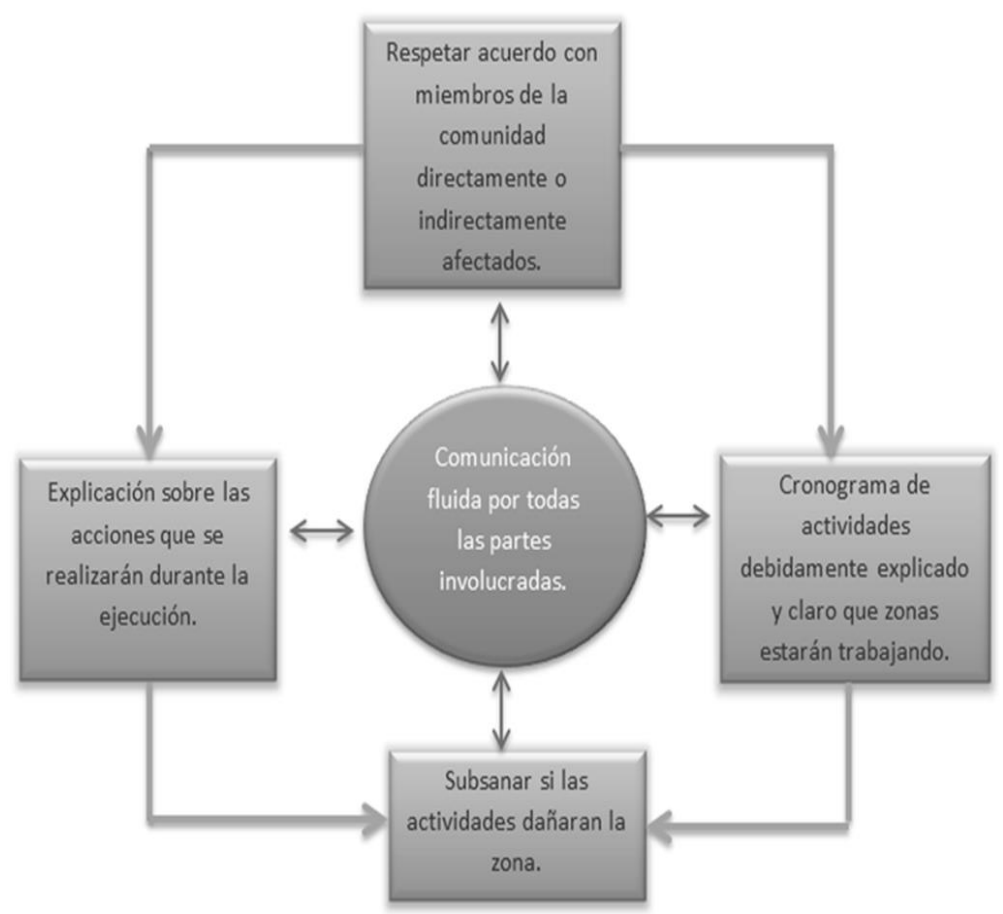

Figura 4. Durante la Construcción de un Proyecto Eólico.

Fuente: Elaboración propia

- Después de la construcción de Proyecto Eólico.

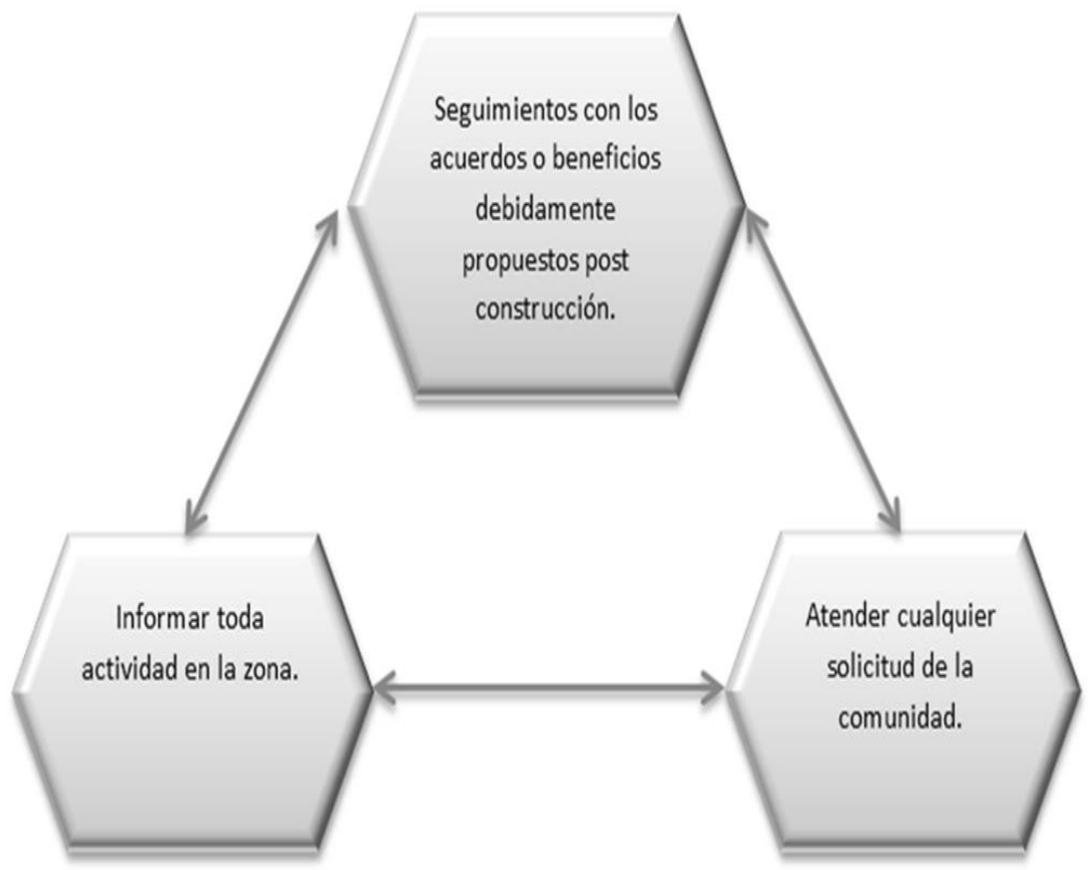

Figura 5. Después de la construcción de Proyecto Eólico. Fuente: Elaboración propia 


\section{Conclusiones}

Es necesario proponer un plan que compatibilice las estrategias de desarrollo económico y social con las de preservación ambiental, asimismo es necesario tener en cuenta un plan de manejo para áreas de conservación natural o zonas arqueológicas. Es importante que con todo ello se incorpore una sostenibilidad de la población. El sector público debe tener una función de orientador, promotor y armonizador de las acciones velando por el desarrollo de la zona que se intervendrá. Es importante tener sistemas de monitoreo y evaluación tanto en el aspecto social, económico, ambiental y legal. Este tipo de proyectos debe tener como objetivo principal el incentivar a la comunidad a trabajar en conjunto para mejorar el desarrollo. Deben implementarse Evaluaciones de Impacto Ambiental (EIA), en todos aquellos proyectos que involucren un impacto significativo sobre el medio ambiente. La honestidad un factor muy importante cumplir lo que se promete. Además de buscar el bien común y que todos los actores tengan mejoras. Este trabajo es una pequeña pieza del rompecabezas, pero teniendo en cuentas estas recomendaciones se tendrá una visión más clara de lo que se busca en cuanto a la socialización de un proyecto eólico. La guía puede servir como un primer paso para considerar la implementación de metodologías de socialización por tecnología, ya que cada tecnología tiene sus particularidades en el impacto, tanto social como ambiental de un proyecto de generación de energía eléctrica con recursos renovables.

Los autores agradecen la colaboración del personal encargado de la socialización del proyecto eólico ubicado en Cerro de Hula, en Honduras.

\section{Bibliografía}

AFE-COHDEFOR, 2015. Anuario Estadístico forestal. (visitado última vez el 30 de mayo del 2017) Disponible en: http://icf.gob.hn/wp-content/uploads/2015/09/Anuario-Forestal2007.pdf

INE, 2012. Estadísticas Nacionales. (visitado última vez el 30 de mayo del 2017) Disponible en: http://www.ine.gob.hn/index.php/25-publicaciones-ine/102-consulta-base-de-datosredatam-cnpv-2013

Flores, W., (2016). El sector energía de Honduras: Aspectos necesarios para su comprensión y estudio, página 79-80, Tegucigalpa, Honduras(P.) ISBN: 978-99926-52-78-7.

Dirección General de Energía, 2016. Proyectos eólicos en operación comercial y de capital privado en Honduras (última vez visitado el 22 de mayo del 2017) Disponible en: http://www.miambiente.gob.hn/

IGWIA, 2010 (última vez visitado el 01 de junio del 2017) Disponible en: http://www.iwgia.org/regions/latin-america/honduras

Honduras, Ley de Propiedad, Decreto No. 82-2004, Capitulo III Del Proceso de Regulación de la Propiedad Inmueble para Pueblos Indígenas y Afrohondureños Artículo 93,94,95,96,101. 
Disponible en:

http://tramites.gob.hn/sites/default/files/Ley\%20de\%20la\%20Propiedad.pdf

Palacios, C., 2007. Pueblos indígenas de Honduras. (última vez visitado el 01 de junio del 2017) http://www.territorioindigenaygobernanza.com/hon_07.html 